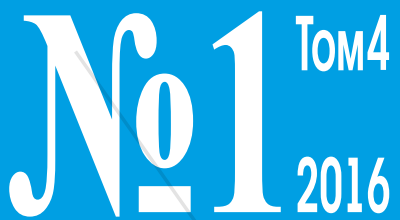

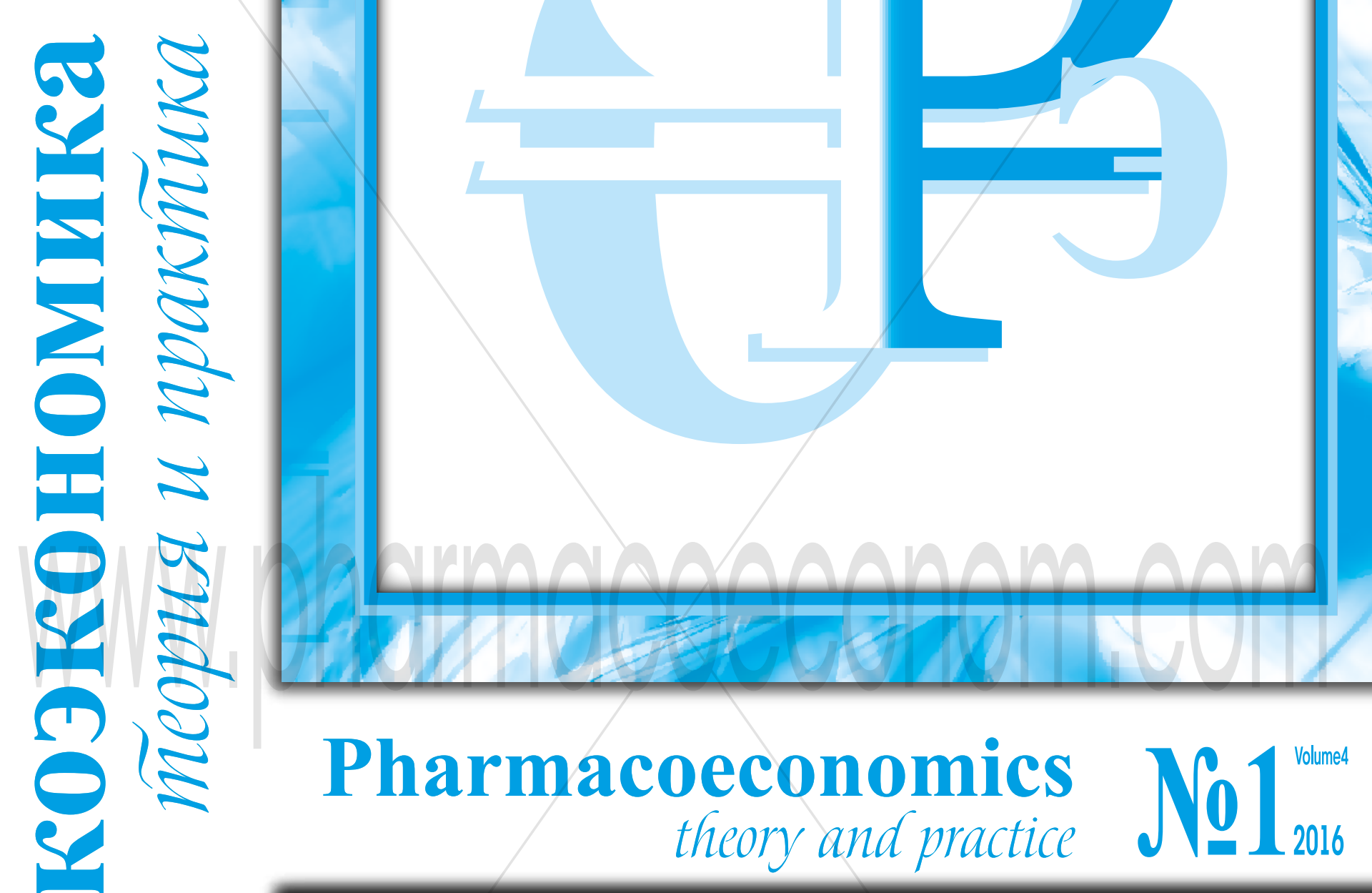

$\square$ МЕТОДОЛОГИЧЕСКИЕ ОСНОВЫ ФАРМАКОЭКОНОМИЧЕСКОГО МОДЕЛИРОВАНИЯ

$\square$ РЕЗУЛЬТАТЫ РОССИЙСКИХ ФАРМАКОЭКОНОМИЧЕСКИХ ИССЛЕДОВАНИЙ

口 МАТЕРИАЛЫ Х НАЦИОНАЛЬНОГО КОНГРЕССА С МЕЖДУНАРОДНЫМ УЧАСТИЕМ «РАЗВИТИЕ ФАРМАКОЭКОНОМИКИ И ФАРМАКОЭПИДЕМИОЛОГИИ В РОССИЙСКОЙ ФЕДЕРАЦИИ» 4-5 апреля 2016 г., г. Нижний Новгород 


\title{
СРАВНИТЕЛЬНЫЙ КЛИНИКО-ЭКОНОМИЧЕСКИЙ АНАЛИЗ ИСХОДОВ САХАРНОГО ДИАБЕТА 2 ТИПА ПРИ ЛЕЧЕНИИ ИНСУЛИНАМИ ГЛАРГИН ИЛИ ДЕГЛУДЕК В СОСТАВЕ БАЗИС-БОЛЮСНОЙ ИНСУЛИНОТЕРАПИИ
}

\section{COMPARATIVE CLINICAL AND ECONOMIC ANALYSIS OF THE OUTCOMES OF TYPE 2 DIABETES MELLITUS (T2DM) TREATED WITH INSULIN GLARGIN OR INSULIN DEGLUDEC AS PART OF BASAL-BOLUS THERAPY}

\author{
Колбин А.С. ${ }^{1,2}$, Чжао B. ${ }^{1}$, Мосикян А.А. ${ }^{1}$, Курылев А.A. ${ }^{1}$, Бальккина Ю.Е. ${ }^{2}$, Проскурин М.А. ${ }^{2}$ \\ Kolbin A.S. ${ }^{1,2}$, Chzhao V. ${ }^{1}$, Mosikyan A.A. ${ }^{1}$, Kurylev A.A. ${ }^{1}$, Balykina Yu.E. ${ }^{2}$, Proskurin M.A. ${ }^{2}$
}

\section{${ }^{1}$ ГБОУ ВПО «Первый Санкт-Петербургский государственный медииинский университет им. акад. И.П. Павлова», г. Санкт-Петербург \\ ${ }^{2}$ ФБОУ ВПО «Санкт-Петербургский Государственный университет», г. Санкт-Петербург ${ }^{1}$ First Pavlov State Medical University of St. Petersburg, Saint Petersburg \\ ${ }^{2}$ St.Petersburg State University, Saint Petersburg}

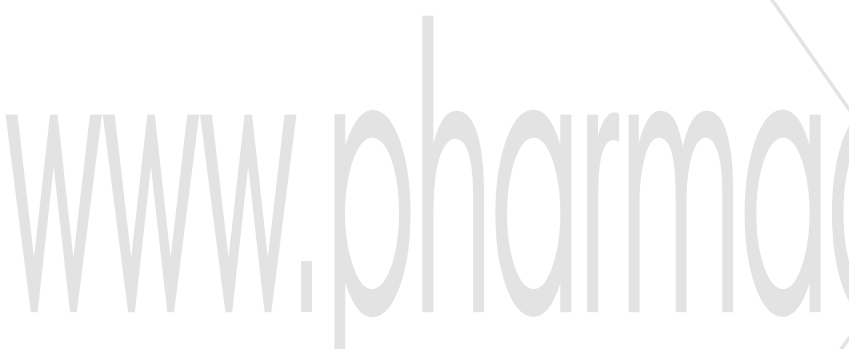

Ключевые слова: клинико-экономический анализ, сахарный диабет 2 типа, инсулин, базис-болюсная инсулинотерапия.

Цель исследования: инсулин деглудек (IDeg) - базальный аналог инсулина сверхдлительного действия, продемонстрировавший в программе клинических исследований 3 фазы возможность достижения такого же эфффективного контроля гликемии у пациентов с сахарным диабетом 2 типа (СД 2), как и при применении инсулина гларгин (IGlar), но при использовании меньших доз (в среднем на 10\%), позволяет существенно снизить частоту подтвержденных гипогликемий (всех - на 27\%, ночных - на $32 \%$ ), а также улучшить оценку ассоциированного со здоровьем качества жизни. Учитывая рост затрат на здравоохранение в условиях ограниченного фринансирования, очень важно сравнить в рутинной клинической практике проффиль «затраты-эффективность» «традиционных» и новых методов лечения. Целью исследования было проведение клинико-экономической экспертизы для оценки возможности сокращения затрат при лечении взрослых с СД 2 при базис-болюсном режиме инсулинотерапии с использованием IDeg.

Материалы и методы: в рамках клинико-экономической экспертизы выполнено построение симуляционной модели исходов при СД 2 на горизонте моделирования 5 лет с использованием пакета MS Excel 2007 для разных режимов базис-болюсной терапии - «инсулин гларгин + инсулин аспарт» и «инсулин деглудек + инсулин аспарт», валидация данных, инкрементальный анализ стратегий инсулинотерапии, анализ чувствительности.

Результаты: стратегия применения IDeg обладает большей клинически значимой эффективностью в отношении снижения гликированного гемоглобина (дополнительное снижение $\mathrm{HbA1c}$ на 0,7 \% после перевода с других базальных аналогов инсулина). Коэффицциенты CER (costeffectiveness ratio), характеризующие эффективность затрат на терапию одного пациента в течение 5 лет для стратегий IDeg и IGlar составили 603215 руб. и 451782 руб. соответственно. При этом соответствующий коэфффициент ICER составил 101236 руб. на одного пациента, что более чем в 10 раз ниже порога готовности общества платить, равного 1341308 руб.

Затраты на тяжелую гипогликемию в течение 5 лет были в 7,67 раза выше для стратегии применения IGlar (226 595 руб.), чем для IDeg (29 556 руб.), а вероятность развития поздних осложнений СД 2 в течение 5 лет при использовании IDeg была ниже (64,71\%), чем при использовании IGlar $(68,91 \%)$.

Стратегия применения IDeg также показала лучшие результаты по сравнению с группой IGlar при рассмотрении в качестве критерия эффективности количества пациенто-лет без осложнений на 100 пациентов (273 пациенто-лет в случае гларгина против 288 пациенто-лет в случае использования деглудека). При рассмотрении данного критерия эффективности коэффициенты CER для стратегий лечения IGlar и IDeg в течение 5 лет были различались незначительно (267 694 руб. и 272149 руб. соответственно). При этом коэффицциент ICER составил 353224 руб. на одного пациента, что в 4 раза ниже порога готовности общества платить. Анализ чувствительности показал, что разница в суммарных затратах между двумя сравниваемыми стратегиями носит случайный характер.

Заключение: терапия IDeg показала большую эффективность в сравнении c IGlar. Инсулин деглудек также обеспечивает большее число пациенто-лет без осложнений. Проведенный анализ чувствительности показал, что для сравниваемых режимов терапии характерны широкие диапазонь прямых затрат, связанные с волательностью входных параметров. Эти диапазоны в существенной степени перекрываются, что свидетельствует о том, что разница прямых затрат, полученная в основном сценарии, между деглудеком и гларгином носит случайный характер при изменении значений входных параметров Коэффиициенты ICER для стратегии IDeg по всем показателям эффективности ниже порога готовности общества платить, что также подтверждает выводы об экономической целесообразности его применения. 